\title{
Mathematical Applications and Modelling in the Teaching and Learning of Mathematics
}

\author{
Jill Brown and Toshikazu Ikeda
}

\section{Introduction}

Applications and modelling have been an important theme in mathematics education during the last 40 years; in particular, through ICMEs regular working/topic groups and lectures on applications and modelling, and the series of International Community on the Teaching of Mathematical Modelling and Applications (ICTMA) conferences, held biennially since 1983. Relations between the real world and mathematics are particularly topical. One reason for learning mathematics is to understand and make sense of the world. The mathematics education community was invited to submit proposals addressing one of six themes and related issues. The focus could be at any level of education including teacher education and the work of mathematicians in the field. It is not surprising therefore that this TSG attracted much attention, with 44 papers submitted. Papers were reviewed by two reviewers. Thirty-six papers were accepted for presentation, from 17 countries (Australia, Austria, Brazil, China, Cyprus, Germany, Israel, Japan, Korea, Mexico, Portugal, Singapore, South Africa, Sweden, Switzerland, UK, USA) and included several teacher authors. Authors received feedback from the co-chairs, and were given time to revise papers in response to this. Also 24 posters related to this TSG (from 10 countries) were presented. Accepted papers were assembled into groups

Organizers Co-chairs: Jill Brown and Toshikazu Ikeda; Team Members: Sung Sook Kim, (Korea), Nicholas Mousoulides (Cyprus), Jussara de Loiola Araújo (Brazil); Liaison IPC member: Morten Blomhoej (Denmark).

\footnotetext{
J. Brown $(\bowtie)$

Australian Catholic University, Melbourne, Australia

e-mail: jill.brown@acu.edu.au

T. Ikeda

Yokohama National University, Yokohama, Japan

e-mail: toshi@ynu.ac.jp

(C) The Author(s) 2015 
for summary, presentation, and discussion. Given the large number of papers, two concurrent sessions were held with participants together initially and for the final discussion. Given space constraints, only presenting authors are named.

\section{Goals and Curriculum}

Paraic Treacy presented The role of mathematical applications in the integration of mathematics and science, using the authentic integration triangle to argue how secondary students in Ireland can be supported to apply their mathematical knowledge to authentic tasks particularly in science contexts. Also looking at secondary school curriculum, Karen Norwood discussed Mathematics instruction using decision science and engineering tools (MINDSET): A multi-step problem solving and modelling course for high school students. She reported on the development and implementations of a year long US curriculum using a problem solving modelling approach. Xiaoli Lu presented a Comparative study on mathematics applications in mathematics textbooks where selected current texts from China and the US were scrutinised for mathematical applications. They report, disappointingly 'most examples in textbooks are traditional mathematical problems without real world contexts'. Jean-Luc Dorier's report on Modelling: a federating theme in the new curriculum for mathematics and sciences in Geneva compulsory education (age 4-15) outlined a new curriculum with modelling as a central theme. However, the definition of modelling was modified from that of Niss, Blum and Galbraith (2007); so rather than modelling involving the extra-mathematical and mathematical domains, although two domains are required, the real world is not an essential one of these.

Jussara Araujo presented Critical construction of mathematical models: An experience on the division of financial resources, reporting on graduate mathematics education students engagement in a critical mathematical modelling task where 'fair criteria' had to be determined to allocate money. The task raised awareness amongst the participants of the importance of modelling. Jung-Ha An reported on Developing mathematical modelling curriculum using difference equations. Examples were shown to demonstrate the use of difference equations in the modelling process in a general mathematics education course. Also at tertiary, Mathematical experiments: A new-designed course for non-mathematical undergraduates in Chinese universities was the focus of Jinxing Xie who shared experiences in designing and teaching courses, for non-mathematics students, on applied mathematics through experiments, modelling and software use. 


\section{Teaching Material, Pedagogy, and Technology}

At the primary level, Nicholas Mousoulides presented Modelling as a bridge between real world problems and school mathematics. He argued for a modelling approach, using engineering MEAs, as a rich source of situations that build on and extend students' existing mathematical learning. Takashi Kawakami presented Necessity for modelling teaching corresponding to diversities: Experimental lessons based on dual modelling cycle framework for the 5th grade pupils. He reported on a teaching experiment with students working on two related tasks. Focussed on teachers of Year 8-9 students, Janeen Lamb presented Planning for building models of situations: What is involved? Data from 8 participants in a project aimed at enhancing teachers' instructional practices were analysed. After completing a modelling activity using an applet, teachers planned how to implement the task in their classrooms. Two studies focused on Year 12 Japanese students: Masahiro Takizawa presented Colors and Mathematics, illustrating how the colour of an image can be used to teach functions and transformations, by adopting a modelling approach. The paper presents a teaching experiment with Year 12 students, using the 'Colors' software. Tetsushi Kawasaki presented A study of mathematical modelling on Year 12 students' function education, reporting the use of modelling in promoting the teaching and learning of two variable functions. The author reports results of a teaching experiment with 15 students.

Issic Leung presented The effect of changing dimensions in illustrative examples in enhancing the modelling process, arguing for a greater emphasis on illustrative examples (e.g., a sketch or diagram). Making greater sense of what is represented should subsequently support mathematical modelling. Also taking a theoretical stance, Vince Geiger presented, On considering alternative frameworks for examining modelling and application activity: The role of texts and digital tools in the process of mathematical modelling, discussing several modelling cycles and frameworks used in either engaging in modelling or by researchers in the field. He argued that models for teaching and learning can be applied to modelling situations. His focus is on the interplay between task, teacher, students and tools.

\section{Experimental Research}

Irit Peled presented More than modelling skills: a task sequence that also promotes children's meta knowledge of modelling, reporting on the development of metaknowledge of modelling by Year 5-6 students as they worked on 10 tasks. Metaknowledge included different ways of mathematising a given problem and hence different models for a single situation can be used appropriately. Focused on Year 5 students Maike Hagena and Rita Borromeo Ferri presented, How do measurement sense and modelling competency influence each other? An intervention study about German middle class students dealing with length and weight. Susanne Grunewald 
presented Acquirement of modelling competencies: First results of an empirical comparison of the effectiveness of two approaches to the development of (metacognitive) modelling competencies of students, reporting use of modelling activities in measurement contexts with Year 8 students. Stanislaw Schukajlow and Andre Krug presented Treating multiple solutions in the classroom and their influence on students' achievements and the affect-The preliminary results of the quasi-empirical study, comparing Year 9 students' work on 'Pythagoras tasks' where no assumptions were required to those where different assumptions and hence multiple solutions were possible, hypothesising the latter leads to better achievement (modelling and intra-mathematical).

Jin Hyeong Park reported on Conceptual understanding of mathematical knowledge through mathematical modelling in a spreadsheet environment. Park sees modelling as representing real phenomena mathematically in order to understand the real world reporting a case study of 15 gifted Year 8 students engaged in an Iced Coffee Task. Findings included development of conceptual calculus understanding and ability to mathematise from their models back to the real world. Also focussing on spreadsheet use, Manfred Borovcnik reported Applications of probability: The Limerick experiments that is, responses of probability workshops participants (inservice secondary teachers), arguing that probability is best taught from a modelling and applications perspective, particularly where technology is used. Here any situation in a classroom is considered as being 'real world'.

Xueying Ji presented A quasi-experimental study of high school students' mathematics modelling competence, reporting modelling competence of Year 10 11 students in China. She found students did not realise the importance of validating their results or critically assessing their models. Milton Rosa presented Ethnomodelling: A research concept on mathematical modelling, arguing the application of techniques in ethnomathematics along with the tools of modelling allows us to see a different reality. Further, research should be from an etic and an emic perspective.

\section{Assessment, Teacher Education, and Obstacles}

Peter Frejd presented Alternative modes of modelling assessment: A literature review, reporting different assessment methods (i.e., written tests, projects) and viewpoints (atomic or holistic). Xenia-Rosemarie Reit and Matthias Ludwig's paper, A crosssection study about modelling task solutions, reported a study where 337 solutions to the Restringing a tennis racket task were analysed. Four main solution approaches were identified. Differences were found in terms of approach taken and progress on the solution path. Kaino Luckson presented The nature of modelling activities and abilities of undergraduate students: some reflections on students' mathematics portfolios, focusing on modelling tasks undertaken by pre-service teacher education students via distance education. Michael Besser reported on Competency-oriented written feedback in every-day mathematics teaching: How to report on students' 
solutions of modelling tasks and how to assess the quality of these reports? This study looked at teacher feedback in the context of technical and modelling tasks, considering strengths/weaknesses of specialized written competency based feedback.

In the field of pre-service teacher education, Thomas Lingefjard presented Learning mathematics through mathematical modelling, arguing that by developing modelling tasks and then engaging in teaching scenarios conceptual understanding occurs. In addition, students came to understand that technology changes what is possible in developing modelling tasks. Dawn $\mathrm{Ng}$ presented, Activating teacher critical moments through reflection on mathematical modelling facilitation where the focus was on the teacher's role and in particular, on the teacher interpretation of student ideas and interventions. The interplay between listening and questioning was critical. Also focused on the role of the teacher was Peter Stender on Modelling in mathematics education development offorms of intervention and their placement in the teacher education and Dominik Leiss Adaptive teacher interventions in mathematical modelling. Both report studies where the balance between student autonomy and teacher interventions was critical.

\section{A Final Word}

There are many interpretations of the terms mathematical modelling and applications. Whilst diversity is desirable, it is helpful to have a common basis for our interpretations. TSG discussion contributes to a shared understanding and the majority of teachers and researchers, see the real world as a critical and essential component of modelling and applications. Following Niss et al. (2007), both mathematical modelling and applications are seen as connecting the mathematical world and the real world. These two worlds are distinct, with the later "describing the world outside mathematics" (p. 3). It is also important to distinguish between modelling and applications. The former begins in the real world and requires a modeller to mathematise the situation, that is, to translate the problem situation into a mathematical situation. In an application, this mathematising has already been done for the solver who works in the mathematical world.

Open Access This chapter is distributed under the terms of the Creative Commons Attribution Noncommercial License, which permits any noncommercial use, distribution, and reproduction in any medium, provided the original author(s) and source are credited.

\section{Reference}

Niss, M., Blum, W. \& Galbraith, P. (2007). Introduction. In W. Blum, P. Galbraith, P., H.-W. Henn \& M. Niss (Eds.), Modelling and applications in mathematics education-The 14th ICMI study (pp. 4-32). New York: Springer. 